\title{
Lab-in-a-Box: Strategies to Teach Online Lab Courses While Maintaining Course Learning Objectives and Outcomes
}

\section{Dr. Kathleen Meehan, Virginia Tech}

Dr. Kathleen Meehan is an associate professor in the Bradley Department of Electrical and Computer Engineering at Virginia Tech. Her previous academic positions were at at the University of Denver and West Virginia University. Prior to moving in academia, she was employed at Lytel, Inc., Polaroid Corporation, and Biocontrol Technology. She received her B.S. in Electrical Engineering from Manhattan College and her M.S. and Ph.D. from the University of Illinois-Urbana/Champaign under the direction of Prof. Nick Holonyak, Jr. Her areas of research include design of optoelectronic materials, devices, and systems; optical spectroscopy; high heat load packaging; and electrical engineering pedagogy.

Mr. Justin Adam Cartwright, Virginia Tech 


\section{Lab-in-a-Box: Strategies to Teach Online Lab Courses While Maintaining Course Learning Objectives and Outcomes}

The Bradley Department of Electrical and Computer Engineering at Virginia Tech has instituted several nontraditional on-campus laboratory courses during the last decade, including the two required circuits labs in the BSEE and BSCpE curricula ${ }^{1}$. The labs utilize a set of equipment known as Lab-in-a-Box (LiaB), which consists of a digital multimeter and a USB-powered oscilloscope with arbitrary function generator as well as a powered breadboard and parts kit. The portable LiaB kit allows the students to perform the labs outside of the classroom. The experiments are designed to reinforce student learning of fundamental concepts and demonstrate complex theorems that are covered in a companion lecture course. The experiments are scheduled so that the students can effectively build upon their theoretical knowledge of circuit analysis, circuit simulation, measurement techniques, and data interpretation as the semester progresses. In addition, the LiaB experiments require the students to perform statistical analysis, program in MATLAB, interpret datasheets, learn/apply debugging techniques, and work on open-ended design projects.

Both of the circuits lab courses initially incorporated a face-to-face lecture prior to the assignment of each experiment. The topics covered in the lecture included an introduction to the experiment with a brief review of the concepts that would be put into practice, instructions on how to run specific simulations using PSpice required to model to operation of the circuit, demonstrations of any new measurements techniques that the students would have to perform, a brief explanation about information that was contained in datasheets that the students would have to use to select the appropriate components for their designs, and comments about common errors in the design, construction, and measurement. The lectures would run about one hour at the beginning of the semester, but would slowly decrease in length as students became more skilled in their experimental techniques and had overcome any issues that they had with the hardware and software.

The lecture was found to provide necessary student-instructor interactions to build student confidence as they conduct experiments on their own and to rapidly address computer-softwarehardware conflicts that students encounter as they use PSpice, MATLAB, and the oscilloscope during the first circuits lab course. However, there were some complicating factors that can negatively influence the value of the lectures. The hardware and software used in the course are unfamiliar to most graduate students who have studied at a different undergraduate institution. The work schedule of a graduate teaching assistant (GTA) assigned to teach the lectures must also include a certain amount of time specifically for the GTA to gain adequate experience with the hardware and software utilized in the course. Furthermore, a laboratory staff member must provide technical support and advise to reduce the GTA's learning curve. While the lack of direct experience with the hardware and software can be addressed, there are more problematic issues. The experimental skills in circuits and electronics of many graduate students are still 
developing and not all of the graduate students in the GTA pool are interested in the subject matter. This lack of experience and interest is much more difficult to overcome, yet is quickly sensed by the undergraduates taking the course who will reflect this in their comments on the quality of instruction at the end of the semester. Thus, the selection of the instructor for the lectures has been a critical factor to the successful introduction of guided self-learning in experimental techniques using LiaB.

\section{Development of online circuits laboratory course for on-campus students}

Motivation: While a physical lecture was also incorporated in the second circuits laboratory course, a majority of students requested its elimination in course exit surveys and student evaluation of instruction. Acting upon this input, the second on-campus lab course has been taught as an online course with lectures and supplemental materials available as a video of voiced-over PowerPoint slides for the past two years. We note that there were a number of aspects of the original LiaB-based course that were kept unchanged as the lecture component of the course was moved online. These include access to face-to-face technical support and demonstration of the operation of the circuits by students to a GTA to receive credit for each experiment.

Learning modules: In moving to an online format, a more complete set of instructional materials has been written and presented in a student-friendly manner. This does not mean that the materials are presented in a 'glossy' format, but that the materials for each experiment are organized into several stand-alone modules. Each module covers one topic and the modules for an experiment are organized so that each module pertained either to issues related to the design of the circuit described in the experimental procedure or to some aspect of one piece of hardware or one of the software packages. These modules are posted, as needed, on Scholar (the electronic course management site) in the following order: overview of the experimental procedure, issues related to component selection or operation, tutorials on a facet of MATLAB and/or an aspect of PSpice that would be used in the prelab design, analysis, and simulations; tutorial on a measurement technique, and a tutorial on a facet of MATLAB that is used in the post-lab analysis of results. The overview of the experiment module is always posted while the other modules are included as needed. In addition, links to the report template and

- Week 5 - Capacitance Multiplier

- Experimental Procedure

- Generating Bode Plots in PSpice

- Report Template

- Validation Instructions

Week 6 - Impedance Matching

- Experimental Procedure

- Transfer Functions in MATLAB

- Report Template

- Validation Instructions

Fig. 1: List of learning online modules created for students in the second circuits lab course and posted on the course Scholar site. 
instructions on how students will validate their experiments are also included. A list of the modules that were made for two experiments used in the Fall 2012 semester is shown in Figure 1. As students had access to all of the modules posted for prior experiments, duplicate modules were not reposted and students were referred back to the old modules if they needed a review of the material. For example, students were asked to generate a Bode plot using PSpice as part of the experiment in Week 5. In Week 6, they derived an equation for the voltage transfer characteristic as a function of frequency and plotted the equation in MATLAB using the transfer function (tf) macro and then generated the Bode plots in PSpice. A learning module on the MATLAB tf macro was available in Week 6 modules on impedance matching; however, the learning module for the Bode plots in PSpice was only listed in the learning modules for the Week 5 experiment on capacitance multipliers. It should be noted that the list of learning modules is not very extensive because the students are not novices, having already taken the first LiaB-based course and their self-confidence in their ability to conduct the experiments is reasonably high. Thus, they are more able, and willing, to conduct independent experimentation than the students in the first LiaB-based lab course.

A detailed description about the topics covered in each module was written in PowerPoint. The PowerPoint slides for segments on hardware or software topics included screenshots of each command. It was important to track each of the commands that were required to perform a particular simulation or measurement, no matter how insignificant it may appear to the person writing the segment. Even undergraduate students who developed some of the tutorials failed to include necessary steps, as they considered them trivial. This can easily be attributed to the amount of experience with the hardware and software these students accumulate while preparing the tutorials. By this time, the students had forgotten what level of support they required when first learning to use the equipment and software. Instead, we found that it was best to track the steps of the person developing the tutorial, using either Camtasia (TechSmith) or the Problem Steps Recorder (PSR) option in Windows, as they performed the measurement or simulation. The person then performed the measurement or simulation a second time to collected high quality screen shots of each step using SnagIt (TechSmith), the Snipping Tool in Microsoft Windows accessories, or the computer's PrintScr key. The images in the recordings and the high quality screen captures were compared to insure that images for all critical steps were saved for inclusion into the learning module. Annotations to the screenshots were included after importing the images into PowerPoint to highlight where mouse clicks were to be done and to explain the step in text. The PowerPoint slides were saved as .pdf files, which the students could modify using PDF Annotator, which they receive in the Freshman Engineering software bundle. Many of the segments have recorded audio to accompany each of the slides. The voiced-over PowerPoint slides are converted to a Flash presentation using Audacity (available at http://audacity.sourceforge.net/) and Presenter (Adobe). The .pdf file and the Flash video are provided to students. From informal surveys of students, about half of the students view only the .pdf files while the others preferred the Flash presentations. Students who use the .pdf files have said that they skim through the file to quickly review the steps that had to be performed and to 


\section{Gyrator}

- Operational Amplifier circuit that has a frequency response similar to an inductor.

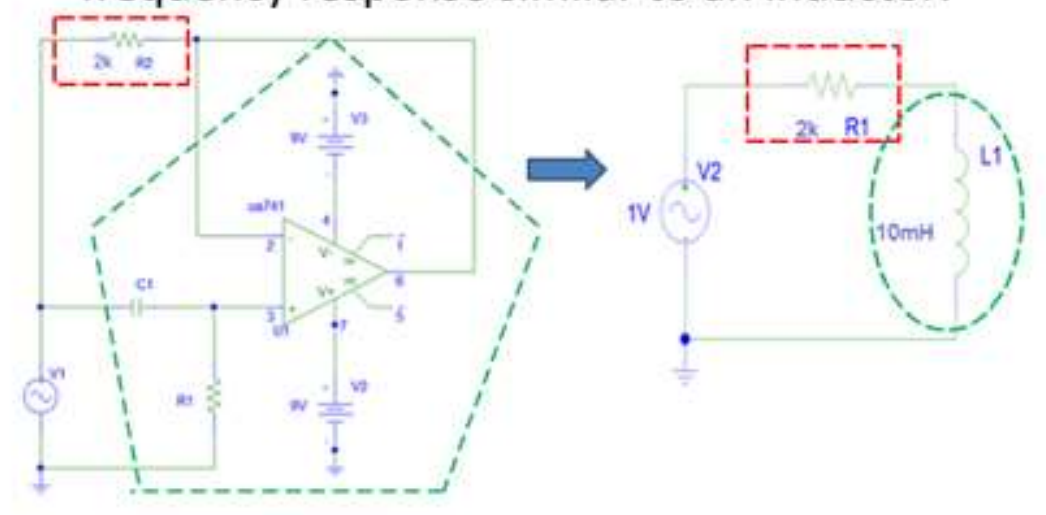

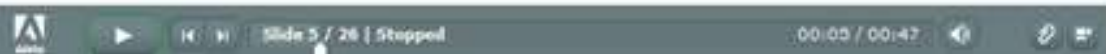

Fig. 2. Screenshot of one of the slides in the Flash tutorial of the online overview of the experiment on the design and operation of gyrators.

read the slides in more detail that describe steps that they didn't realize that they had to do after they attempted the process themselves. One student commented that the reason why he preferred the Flash presentations was because he could launch the video as he started to perform the measurement that was described in the video. He could insure that he carried out the measurement properly by listening to the steps as he performed them himself. Presenter was used rather than Camtasia because an index of all of the titles of the PowerPoint slide in the video (on the right in Fig. 2) was automatically generated and available to students so that they could easily select an individual slide to repeat the voice-over of that particular slide.

Conclusions drawn from the online lab course for on-campus students: No significant differences have been noted in exit surveys before and after the change in lecture format. Only 2 of the more than 225 students enrolled in the second circuits lab course suggested in the exit surveys that physical lectures be re-instituted. This is not surprising as most students have already completed the first LiaB- based lab course, a traditional in-class electronics course in which PSpice is used extensively, and an introductory course in computer engineering in which they are assigned five hands-on projects that are also completed outside of a classroom. The only students who may be at a disadvantage are transfer students. However, these students generally take the second circuits lab course during their second semester at Virginia Tech and have learned PSpice and many of the measurement techniques before enrolling in the course. In addition, tutorials on the use of the oscilloscope, PSpice and MATLAB that were written for the 
first circuits course are posted on the Scholar site for the second circuits course and can be accessed at any time by the transfer students as well as any student who requires a review.

Development of the second online laboratory course for online students: For the summer 2011 semester, it was originally planned to move the first circuits course to an online format while offering the companion lab course as an on-campus course with a physical lecture. However, due to low enrollment, only the online lecture course was taught. The format for course delivery returned to normal during the following academic year. In summer 2012, both the circuits lecture and lab courses were offered online. Of the 12 students enrolled in the lab course, 10 were also enrolled in the online lecture course, 1 student was a transfer student who had received credit for a lecture course taken at another institution, and 1 student was retaking the lab. More than half of the students were living away from campus while taking the course.

While most of the experiments were similar to those assigned during the spring semester, there were some differences in assignments, make-up policies, and grading schemes. In addition, an automated grading program, tested in spring 2012 for half a semester, was used extensively during the summer semester. There were two significant differences between the on-campus and online first LiaB-based lab course. First was the volume of learning modules that had to be written to support the students. The second was the method used to provide live technical support as the students conducted the experiments to validate the circuits constructed by the students and to verify that the students were performing the measurements using the proper techniques.

Learning modules: There were numerous supplemental learning modules that had to be written to augment the material that had already been developed for the lectures given for the on-campus as the course was moved online. While the existing lecture materials contained the information that would become the overview of the experiments, the learning material on measurement techniques and circuit simulations had always been delivered as hands-on demonstrations by the instructor using real equipment and components or by launching PSpice to run the simulation real-time. Students in the on-campus course had been encouraged to bring their own equipment and computers so that they could perform the same measurements and simulations during the demonstrations. However, none of that material existed in an electronic format. Therefore, extensive instructions on PSpice and the operation of the scope and DMM were written to support every experiment assigned because there were new experimental techniques and/or simulations introduced each week. Additional supplemental materials were also written to provide answers to questions that were commonly answered during the lectures and to address issues that arose as the online course was taught. Following the format used to present the online learning materials to students in the second circuits course, single-topic modules were organized on the course Scholar site in the order in which the topics were covered in each of the experimental procedures (Fig. 3). 
- Week 6 - Experiment 12

- Lecture

- Velleman Function Generator

- Velleman Oscilloscope

- Report Template

- Instructions

- Week 7 - Experiment 14

- Lecture

- Measurement of Capacitance

- Exporting Scope Data to Excel

- Report Template

- Instructions

Fig. 3: List of learning online modules created for students in the first circuits lab course as taught online and posted on the course Scholar site.
- PSpice Simulation with Square Wave Sources

Real-time personal student-instructor interactions: The second issue that was unique to this online course was the need to have real-time visual interactions with the students to observe their experimental techniques and to provide the 'face-to-face' engagement that had been found to support the development of student confidence during the on-campus course. As mentioned in the description about the selection of the oncampus lecturer, it was critical that the GTA have extensive experience in electronic design and was familiar with the equipment in LiaB as well as the software packages used in the experiments for the on-campus course. This was even more important for the online course. A graduate teaching assistant (GTA) who had assisted with the on-campus lab course for a year and had a strong background in analog electronic design and measurement was asked to support both the online laboratory and lecture course, which allowed the GTA to refer students back to the appropriate lectures on concepts and analytical techniques that the students were in the process of learning when the students ran into problems putting them into practice in the experiments.

The GTA held regularly scheduled hours on Skype, a program that had been evaluated for this application in a previous $s^{2}{ }^{2}$, to answer questions related to the assigned experiments. Students signed up to be contacted by the GTA to ask questions about measurement techniques or their PSpice simulations, or to ask for a review of their hand calculations. The sign-up process using an electronic board at https://secure.hosting.vt.edu/www.opel.ece.vt.edu/login.php, which allowed the GTA to allocate his time during his office hours and enabled students to see how many others were in line before them. The screen share function on Skype allowed the GTA to see the schematics that the students had constructed in PSpice as well as inked calculations written on their tablet computers. This was critical to his ability to provide advice on how to correct errors in the PSpice schematics, in the students' calculations, or in the logical approach that they were using to solve the design problem. Most students were able to demonstrate their circuits live to the GTA using the video call function on Skype. In one case, a student posted YouTube videos in which he performed the required measurements listed in the Instructions file that was posted with the other learning modules for each experiment (see Fig. 3) as his internet access speed prevented real-time video conferencing. 
Conclusions drawn from the online lab course for online students: Student performance in the online course, the percentage of students that completed the course, and the distribution of final grades could not be compared in a statistically meaningful way, given the small student population in the online course. Qualitatively, there was little difference between the learning outcomes achieved by the end of the online course by the students who successfully completed the course as compared to those students who successfully completed the on-campus course. The percentage of students that withdrew from the course or failed to complete the course was slightly higher than would be expected in the on-campus course, but was not out-of-line with the percentages of students who did not complete or failed the companion online lecture course or other on-campus summer session courses.

Student confidence in their likelihood of success and achievements in pre- and post-course surveys, respectively, were almost universally higher than levels measured in students during spring 2012. The authors speculate that this is correlated with the personality traits required to elect to participate in the first offering of an online lab course. Given these results, the department has decided to offer this online circuits course again in Summer 2013.

\section{Acknowledgements}

The authors acknowledge the support of the Bradley Department of Electrical and Computer Engineering at Virginia Tech and the National Science Foundation (Award 0817102). We also wish to acknowledge the contributions of several individuals on wrote some of the instructional materials used in the first circuits course - Dr. Richard L. Clark, Jr. (Virginia Western Community College), Ms. Justeen Olinger, and Mr. David Fritz.

\section{Bibliographic Information}

${ }^{1}$ Hendricks, R.W., K-M. Lai, and J.B. Web (2005). "Lab-in-a-Box: Experiments in Electronic Circuits That Support Introductory Courses for Electrical and Computer Engineers." Proc. ASEE Annual Meeting, June 12-15, 2005, Portland OR. (available online at www.asee.org).

${ }^{2}$ K. Meehan, J. Quesenberry, J. Olinger, K. Diomedi, R.L. Clark, R.W. Hendricks, and P.E. Doolittle, "Hands-on Distance-Learning Laboratory Course Using Internet Video Tools", Proc. ASEE Annual Meeting, June 14-17, 2010, Louisville, KY. (available online at www.asee.org). 